\title{
A Comparison between Neural Network Methods for Learning Aggregate Functions
}

\author{
Werner Uwents ${ }^{1}$ and Hendrik Blockeel ${ }^{1,2}$ \\ ${ }^{1}$ Department of Computer Science, Katholieke Universiteit Leuven \\ ${ }^{2}$ Leiden Institute of Advanced Computer Science, Leiden University
}

\begin{abstract}
In various application domains, data can be represented as bags of vectors instead of single vectors. Learning aggregate functions from such bags is a challenging problem. In this paper, a number of simple neural network approaches and a combined approach based on cascadecorrelation are examined in order to handle this kind of data. Adapted feedforward networks, recurrent networks and networks with special aggregation units integrated in the network can all be used to construct networks that are capable of learning aggregate function. A combination of these three approaches is possible by using cascade-correlation, creating a method that automatically chooses the best of these options. Results on artificial and multi-instance data sets are reported, allowing a comparison between the different approaches.
\end{abstract}

\section{Introduction}

Instead of using the classical attribute-value representation, it is more natural in some application domains to represent data as bags of vectors. This means that each data instance is described by a bag of vectors, but has only one target vector. Different instances can have different numbers of vectors in their bags, making traditional machine learning methods that have a fixed number of inputs impractical to use for this type of data. Moreover, the ability to process bags can be seen as the crucial element that distinguishes attribute-value methods from relational approaches, as explained in [1]. If we look at the kind of features that are constructed by a relational learner, an essential property of these features is that they map sets or bags of objects to a single scalar value. Functions that perform this kind of mapping are called aggregate functions.

Typically, systems that are capable of learning this kind of aggregate features use some simple and predefined aggregate functions like max or count. Most often, a propositionalization approach is followed. In this kind of approach, each data element is summarized into a vector of fixed length before the actual learning process. The components of this vector are then used as features. The aggregate features can become more complex when nesting selection conditions is allowed. As has been pointed out by Blockeel and Bruynooghe [1, the features constructed by relational learning systems are generally of the form $\mathcal{F}\left(\sigma_{C}(S)\right)$ with $S$ a set of objects, $C$ a condition defined over single objects, $\sigma$ the selection operator from relational algebra, and $\mathcal{F}$ an aggregate function, which maps a 
set to a scalar. But to keep the propositionalization approach feasible, a limited set of $\mathcal{F}$ functions needs to be used, and the number of different $C$ considered for $\sigma_{C}$ must remain limited. For instance, Krogel and Wrobel 2] allow a single attribute test in $C$, but no conjunctions.

Propositionalization is not the only possibility however. Some systems allow to learn aggregate functions in a more flexible and direct way. For instance, in ILP, if we have a clause happy_father $(X)$ :- $\operatorname{child}(Y, X)$, the "feature" constructed is essentially of the form $\exists y: \operatorname{child}(y, x)$, which tests if the set of all $y$ 's related to $x$ through the child relation is empty or not. In the above clause, $S$ is the set of children of $x, C$ is true, and $\mathcal{F}$ is the "there exists" operator $(\exists)$. For the clause happy_father $(X)$ :- $\operatorname{child}(Y, X)$, age $(Y, A), A<12, S$ and $\mathcal{F}$ are the same, while $C$ is a condition on the age of the children now. But a direct approach has disadvantages as well. The structure of the search space of features of the form $\mathcal{F}\left(\sigma_{C}(S)\right)$ becomes much more complex and difficult to search when $\mathcal{F}$ can be something else than $\exists[3$. Blockeel and Bruynooghe 1 pointed out that for this reason these systems typically construct structurally complex conditions but always use the same, trivial aggregate function, namely $\exists$. The importance of using more complex aggregate functions has however been recognized by many people [2]4.

Therefore it is useful to study how direct approaches could include aggregate functions other than $\exists$. More recently, methods for learning more advanced features of the form $\mathcal{F}\left(\sigma_{C}(S)\right)$ have been proposed. Vens, Van Assche et al. [5]6] proposed a random forest approach that avoids the problems of searching a complex-structured search space, while Vens $[3$ studied the monotonicity properties of features of the form $\mathcal{F}\left(\sigma_{C}(S)\right)$ and showed how efficient refinement of such features is possible for the most commonly occurring aggregate functions.

All these are symbolic approaches to learning aggregate functions. In parallel, Uwents and Blockeel studied to what extent subsymbolic representations of aggregate features can be learned using neural network approaches. The advantage of this approach is that the complex search is avoided and that the function $\mathcal{F}$ and condition $\sigma_{C}(S)$ in the $\mathcal{F}\left(\sigma_{C}(S)\right)$ feature are learned simultaneously and automatically. Recurrent neural networks were first proposed for this task, leading to the concept of relational neural networks 7 . While a regular network maps one input vector to an output vector, recurrent networks can map a sequence of input vectors to a single output vector. This property was exploited to handle sets of vectors, the elements of which were given as input to the network in random order. Recurrent networks are however not the only option for learning aggregate functions, a number of other network structures could be used as well. Recently, also a cascade-correlation approach has been proposed [8].

All the previous work focused on extending relational learning with the ability to learn aggregation functions, and evaluating how this ability affects overall predictive accuracy. In this work, however, we focus on the particular problem of learning aggregate functions of the form $\mathcal{F}\left(\sigma_{C}(S)\right.$ ), with $S$ a given set of tuples. From the point of view of relational learning, this is a simplified setting: there is only one 1- $n$ relation, relating each single target to a single bag of tuples. 
All these tuples are of the same type, and they do not participate in further relations. While this is a limitation from the point of view of relational learning, the exclusion of such further relational information allows us to get a better view on the inherent capacity of certain neural network structures to learn particular aggregate functions, which is the main aim of this research. Another point of difference with previous work is that we study a broader range of aggregate functions than has previously been done.

We will compare different methods for subsymbolic learning of aggregate functions from bags of vectors with an associated target value. In Section 2, we discuss three basic and simple methods that use adapted forms of standard neural network structures, as well as a combined approach using cascade-correlation. In Section 3, we present experimental results comparing the different approaches to each other. We conclude in Section 4.

\section{Neural Network Methods}

\subsection{Simple Methods}

There are some straightforward and rather simple ways to use standard neural networks structures in a slightly modified form to process bag data and learn aggregate functions. Three of these simple approaches are discussed here.

Adapted Feedforward Networks. Probably the simplest way to extend normal feedforward neural networks to bags, is to use a feedforward network with $m_{\max } \times n$ inputs. In this formula, $m_{\max }$ is the maximum number of vectors a bag can contain, while $n$ is the number of real numbers per vector. When a bag of size $m$ is fed into the network, the first $m \times n$ inputs are filled with the values of the $m$ vectors in the bag. The order in which this happens, is not important. The remaining $\left(m_{\max }-m\right) \times n$ inputs are filled up with padding values. For these padding values, a fixed, given value is used.

One of the disadvantages is that $m_{\max }$ can be quite large. This results in a network with a lot of connections and corresponding weights to train, which is bad because it increases the complexity of the model and the danger of overfitting. Another disadvantage could be that the number of hidden neurons is fixed. It seems more logical that the number of hidden neurons should be somehow proportional to $m$, the cardinality of the input bag, because the complexity of the function is probably also proportional to the size of the bag. These disadvantages can be countered by duplicating the hidden neurons for each input vector in the bag and by sharing the weights. Because the function that has to be learned by the network should be invariant to permutations of the input vectors, a lot of weights can be shared across the network. More precisely, the hidden units are copied for each input vector and the weights are shared between these copies. The weight sharing also applies to the weights for the different input vectors within a copy. Finally, also the weights for the connections between these copies and the output units are shared. This network structure will be referred to as sym because of the symmetry introduced by the weight sharing. 
Networks with Aggregation Units. It is also possible to add some predefined aggregate functions to the network. They are placed after a neuron. Activation values for this neuron are computed for all input vectors in the bag and then the aggregate function is used to combine all these activation values into a single value. The aggregate functions could be placed after the hidden or the output units. Typical functions would be sum or max. The only condition for these aggregate functions is that they have to be derivable to be able to compute the gradient. This kind of approach has been applied to multi-instance problems before, where a softmax function was used 9 . The softmax function is defined as

$$
\frac{1}{M} \log \left(\sum_{i=1}^{n} e^{M \cdot x_{i}}\right)
$$

where $M$ is a constant and the larger the value of $M$, the closer this function will approximate the real max function. Instead of choosing a value for $M$ that is large enough to give a good approximation, one could take the limit for $M$ going to infinity, which gives the real max function and a formula for its derivative. The sum, softmax and max function will be considered here as possible aggregate functions. Note that the use of a specific kind of aggregate function in the network, does not necessarily limit the network to learning only this kind of aggregate function from the data. By learning the right weights, other aggregate functions could still be approximated. The different network types will be referred to as sum, hsum, smx, hsmx, max and hmax. The prefix $h$ indicates that the function is placed after each hidden unit, otherwise they are placed after the output units.

Recurrent networks. Another possibility is to use recurrent networks. They are typically used to process time series or other sequences of data, but they could be used to process bags as well, as done in [7]. The $m$ vectors of the input bag are fed into the network one after another. For each vector, the activation levels of the hidden neurons are updated. After processing all input vectors, the last activation levels are used to compute the activation level of the output neuron. This kind of approach could be problematic if applied to large bags because recurrent networks are hard to train on large sequences. In the rest of the paper, two types of recurrent networks will be considered, locally and fully recurrent networks. In the locally recurrent networks, denoted as $l r c$, each hidden unit has a recurrent connection only with itself. In fully recurrent networks, named frc, each hidden unit also has recurrent connections with all other hidden units.

\subsection{Combined Method}

The previously discussed network structures have two important drawbacks. First of all, one of the network types has to be selected. After that, also the number of units in the hidden layer has to be chosen. Both choices are not trivial. A cascade-correlation approach solves this problem and makes a combination of the simple methods possible. The idea behind the original cascade-correlation 


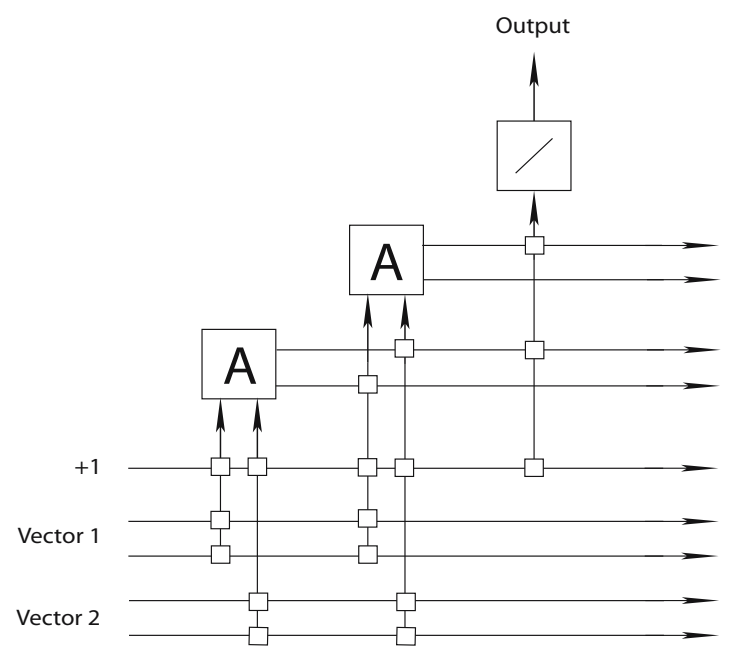

Fig. 1. Schema for an aggregate cascade-correlation network with 2 input vectors

algorithm 10 is to learn not only the weights, but also the structure of the network at the same time. This is done in a constructive way, meaning that only one neuron at a time is trained and then added to the network. One starts with a network without any hidden unit, and then hidden neurons are added, one by one, until some stopping criterion is satisfied. Once a hidden neuron has been added to the network, its weights remain fixed throughout the rest of the procedure. This also means that, besides the actual input vector, the output values of these existing hidden units can be used as extra inputs for any new hidden neuron. At the output, a linear function can be used.

The training of the network is done in two alternating phases. Before a new hidden neuron is added, its weights are trained while keeping the weights of all other hidden units fixed. This training is not done by minimizing the squared error between target and output, but by maximizing the correlation with the residual error. The residual error is defined as the difference between the actual target value and the output of the existing network, before adding the new neuron. The assumption is that its correlation with the residual error will make a new neuron useful in reducing the residual error and improving the prediction of the actual target. The maximization is done by computing the gradient and performing some form of gradient ascent. Instead of training only one candidate neuron at a time, a pool of neurons, initialized with random weights, can be trained. At the end, the best one is selected. This increases the chance that a good candidate will be found. Once the best candidate is selected and added to the network, the output weights for the updated network can be trained. If a linear function is used at the outputs, the output weights can be obtained by simple linear regression.

The concept of cascade-correlation networks can be extended to networks for learning aggregate functions, as proposed in 8. The crucial difference is that 
instead of the simple hidden neurons, units that can process bags are used. These units come from the simple networks presented above. The sym, hsum, hmax, $h s m x$ and lrc units can all be used as aggregation units in the hidden layer of the cascade-correlation network. For the rest, the network and the training of it works in the same way as for the feedforward cascade-correlation networks. A schema of an aggregate cascade-correlation network for 2 input vectors is shown in figure 1

With all parts of the aggregate cascade-correlation network explained, it only remains to discuss the training of the network in more detail. Each time a new unit should be added to the hidden layer, a pool of units is created of all possible types. Weights are initialized randomly. After that, all units in the pool are trained for a number of iterations, similar to backpropagation. This training is basically a gradient ascent, maximizing the correlation with the outputs. The computation of the gradient depends of course on the type of unit. The gradient ascent itself is actually done by using resilient propagation, as described in [11. This method has the advantage that the step size is determined automatically and convergence is faster than for a fixed step size. The basic idea is to increase the step size when the sign of the gradient remains the same, and decrease the step size when the sign changes.

When all units in the pool have been trained, the best one is chosen. In this case, the best unit is the one with the highest correlation. When the unit with the highest correlation has been chosen, it is installed in the network and the output weights have to be learned again. Because linear activation functions are used for the output units, the output weights can be determined with least squares linear regression.

\section{Experiments}

In this section, a number of experimental results will be discussed. First, a series of experiments is carried out on artificially created data sets. After that, the methods are evaluated on a multi-instance data set.

\subsection{Simple Aggregates}

A simple experiment to examine the capacity of the aggregate cascadecorrelation network, is to create artificial data with predefined aggregate functions and train the networks on it. The data consists of bags with a variable number of elements. Each element of the bag is a vector with five components. Only the first or the first and second component are relevant for the target value, depending on the aggregate function under consideration. The values of these components are randomly generated, but in such a way that the target values are uniformly distributed over the possible target values. All the other components are filled with uniformly distributed random numbers from the interval $[-1,1]$. It is very likely that the number of vectors in the bags influences the difficulty of the learning task, so different sizes are tested. The data sets denoted as small 
contain 5 to 10 vectors per bag, the medium data sets 20 to 30 and the large ones 50 to 100. Each data set contains 3000 bags. A range of different aggregate functions are used to construct the data sets:

1. count: the target is the number of vectors in the bag.

2. sum: the target is the sum of all values of the first component of the bag vectors.

3. max: the target is the maximum value of the first component of the bag vectors.

4. avg: the target is the average value of the first component of the bag vectors.

5. stddev: the target is the standard deviation of the values of the first component of the bag vectors.

6. cmpcount: the target is the number of bag vectors for which the value of the first component is smaller than the value of the second component.

7. corr: the target is the correlation between the first two components of the bag vectors.

8. even: the target is one if the number of positive values for the first component is even, and zero if it is odd.

9. distr: the target is one if the values of the first component come from a gaussian distribution, and zero if they are from a uniform distribution.

10. select: the target is one if at least one of the values of the first component lies in a given interval, and zero otherwise.

11. conj: the target is one if there is at least one vector in the bag for which the the first and the second component lie in a certain interval.

12. disj: the target is one if there is at least one vector in the bag for which the first or the second component fall in a certain interval.

The first 7 data sets have a numerical target, the others a nominal target. In case of a nominal target, the number of positive and negative examples are equal. Experiments are done using five-fold cross-validation. For each fold, 1800 examples are used as training set, 600 as validation set and another 600 as test set. For the simple approaches, the number of hidden units is 3 and resilient propagation [11] was used to train the networks for 1000 iterations. For the cascade-correlation method, the maximum number of hidden units is limited to 10 . The number of candidate units trained in every step is 20. Each unit is trained for 500 iterations, which should be enough to have converged to optimal weights. For the data sets with nominal target, the accuracy is reported and for the sets with numerical targets the mean squared error is used. For the simple methods, only the best results are given and it is also indicated which types of network achieved this performance or a performance very close to it. The results are summarized in tables [1] and 2.

From the results, it is clear that most functions can be learned quite well. Only the even function seems impossible to learn. This is not very surprising, given that this is a kind of parity problem, which is known to be difficult. For the distr function, the number of vectors must be large to be able to learn it well. This makes sense because it is easier to say whether a bag of values come 
from a normal or uniform distribution if the bag is larger than when it is rather small. Table 1 also indicates which types of networks are the best to learn the considered concepts. There is no type that is best for all of them. Sometimes it is clear why certain types are the best, for instance for the sum and max data sets, but for other data sets it is not that obvious. This makes it hard to make the right choice beforehand. Furthermore, the results for the cascade-correlation network are always very close to the results for the best simple network or even beat this result. This shows that using the combined cascade-correlation approach is indeed a good idea.

\subsection{Trains}

The trains data sets are also artificially created data sets containing a number of trains. Every train consists of a number of cars, carrying some load. Some of the trains are eastbound, the others are westbound. The direction of the trains is what has to be learned and this target concept is based on the properties of the cars of a train and their loads. The cars of the train constitute a bag for each train. A data generator 12 for this train problem was used to create 12 data sets with different properties. Sets 1 to 4 consist of short trains, having 2 to 6 cars. Data sets 5 to 8 are similar to sets 1 to 4 , except that they contain longer trains. Each of these trains consists of 20 to 29 cars. The used concepts are the same as for sets 1 to 4 , except that the numbers in the aggregations are adapted to the longer length of the trains. Data sets 9 to 12 contain noisy data. This means that a number of samples have been mislabeled. The twelve datasets are defined as follows:

Table 1. Results for the simple aggregate data sets using the simple network structures. Accuracies or mean squared errors are given together with the standard deviations for five-fold cross-validation. The results are those for the best type of network. The types of network that produced this result or very similar results, are indicated in the last column.

\begin{tabular}{|c|c|c|c|c|c|}
\hline & & small & medium & large & type \\
\hline \multirow{7}{*}{$\begin{array}{l}\text { Ind } \\
\text { S2 }\end{array}$} & count & $0.00(0.00)$ & $0.00(0.00)$ & $0.00(0.00)$ & hsum, sym \\
\hline & sum & $0.00(0.00)$ & $0.00(0.00)$ & $0.02(0.01)$ & hsum, sym \\
\hline & $\max$ & $0.00(0.00)$ & $0.00(0.00)$ & $0.01(0.01)$ & (h) $\max ,(h) \operatorname{smx}, \operatorname{lrc}$, frc \\
\hline & avg & $0.03(0.03)$ & $0.01(0.01)$ & $0.01(0.01)$ & $\max , \mathrm{smx}, \operatorname{lrc}$, frc, sym \\
\hline & stddev & $0.02(0.01)$ & $0.01(0.01)$ & $0.01(0.01)$ & hmax, hsmx \\
\hline & cmpcount & $0.00(0.00)$ & $0.00(0.00)$ & $0.00(0.00)$ & sym, hsum \\
\hline & corr & $0.05(0.04)$ & $0.04(0.02)$ & $0.01(0.01)$ & sym, hsum, hmax, hsmx \\
\hline \multirow{5}{*}{ 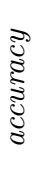 } & even & $52.6(0.0)$ & $51.7(0.6)$ & $51.4(0.5)$ & all types equal \\
\hline & distr & $63.3(0.6)$ & $74.6(0.9)$ & $80.3(0.9)$ & hmax, hsmx \\
\hline & select & $99.6(0.3)$ & $99.9(0.1)$ & $99.8(0.2)$ & $\operatorname{smx}$ \\
\hline & conj & $99.9(0.0)$ & $99.9(0.0)$ & $94.5(0.4)$ & $\max , \operatorname{smx}$ \\
\hline & $\operatorname{disj}$ & $89.3(1.2)$ & $76.7(0.9)$ & $74.6(1.3)$ & hsmx \\
\hline
\end{tabular}


Table 2. Results for the simple aggregate data sets using cascade-correlation. Accuracies or mean squared errors are given together with the standard deviations for five-fold cross-validation.

\begin{tabular}{|c|c|c|c|c|}
\hline & & small & medium & large \\
\hline \multirow{7}{*}{$\begin{array}{l}\text { 程 } \\
\frac{2}{4}\end{array}$} & $\overline{\text { count }}$ & 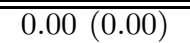 & $0.00(0.00)$ & $\overline{0.00(0.00)}$ \\
\hline & sum & $0.00(0.00)$ & $0.00(0.00)$ & $0.00(0.00)$ \\
\hline & $\max$ & $0.00(0.00)$ & $0.00(0.00)$ & $0.00(0.00)$ \\
\hline & avg & $0.06(0.06)$ & $0.01(0.01)$ & $0.01(0.01)$ \\
\hline & stddev & $0.02(0.02)$ & $0.01(0.01)$ & $0.00(0.00)$ \\
\hline & cmpcount & $0.00(0.00)$ & $0.00(0.00)$ & $0.00(0.00)$ \\
\hline & corr & $0.04(0.04)$ & $0.02(0.02)$ & $0.01(0.01)$ \\
\hline \multirow{5}{*}{ 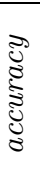 } & even & $52.33(0.00)$ & $50.10(0.73)$ & $51.90(0.72)$ \\
\hline & distr & $66.18(0.56)$ & $76.68(0.79)$ & $84.10(1.57)$ \\
\hline & select & $99.15(0.41)$ & $99.63(0.15)$ & $98.87(0.48)$ \\
\hline & conj & $100.00(0.00)$ & $99.92(0.11)$ & $99.93(0.20)$ \\
\hline & disj & $99.00(0.43)$ & $97.75(0.55)$ & $96.30(1.81)$ \\
\hline
\end{tabular}

1. Trains having at least one circle load are eastbound, the others are westbound.

2. Trains having at least one circle or rectangle load and at least one car with peaked roof or 3 wheels are eastbound, the others are westbound.

3. Trains having more than 8 wheels in total are eastbound, the others are westbound.

4. Trains having more than 3 wheels in total and at least 2 rectangle loads and maximum 5 cars are eastbound, the others are westbound.

5. Same concept as for set 1 .

6. Same concept as for set 2 .

7. Trains having more than 53 wheels in total are eastbound, the others are westbound.

8. Trains having more than 45 wheels in total and at least 10 rectangle loads and maximum 27 cars are eastbound, the others are westbound.

9. Same concept as for set 1 , but with $5 \%$ noise.

10. Same concept as for set 1 , but with $15 \%$ noise.

11. Same concept as for set 3, but with $5 \%$ noise.

12. Same concept as for set 3 , but with $15 \%$ noise.

Each data set contains 3000 instances. Experiments are done using five-fold cross-validation which means that for each fold 1800 instances are used for training, 600 for validation and 600 for testing. For the simple approaches, the number of hidden units is 5 and the number of training iterations is 1000 . For the cascadecorrelation method, the maximum number of hidden units is limited to 10 . The number of candidate units trained in every step is 20. Each unit is trained for 500 iterations. The results are given in table 3. It is clear that most concepts can be learned well. Most of the data sets without noise have an accuracy very close 
to $100 \%$. Only for set 8 , which has the most difficult concept, is it impossible to get close to perfect accuracy. For the data sets with noise, the best accuracies are all close to $100 \%$ minus the percentage of noise. It is also clear that cascadecorrelation has a performance that is superior to any of the simple methods, just as for the simple aggregate data sets.

Table 3. Accuracies using five-fold cross-validation for the trains data sets

\begin{tabular}{|r|c|c|c|c|c|c|c|c|c|c|}
\hline & casc-corr & sym & lrc & frc & sum & hsum & max & hmax & smx & hsmx \\
\hline \hline 1 & 99.9 & 99.5 & 96.9 & 100.0 & 83.8 & 99.7 & 100.0 & 100.0 & 99.7 & 100.0 \\
2 & 100.0 & 99.3 & 97.7 & 96.8 & 77.3 & 97.9 & 94.5 & 99.5 & 94.6 & 99.6 \\
3 & 100.0 & 99.9 & 96.0 & 98.9 & 55.0 & 100.0 & 65.3 & 83.6 & 69.1 & 84.7 \\
4 & 98.7 & 98.7 & 81.3 & 94.7 & 78.6 & 88.0 & 86.5 & 77.0 & 86.1 & 81.0 \\
5 & 99.7 & 88.4 & 81.1 & 92.5 & 56.8 & 99.2 & 94.9 & 87.2 & 98.0 & 86.4 \\
6 & 99.4 & 86.9 & 68.8 & 73.9 & 54.7 & 83.1 & 72.7 & 69.4 & 70.0 & 78.5 \\
7 & 99.4 & 97.3 & 51.4 & 51.3 & 52.3 & 98.5 & 56.5 & 62.4 & 57.8 & 63.0 \\
8 & 84.5 & 77.5 & 65.2 & 71.0 & 55.3 & 79.2 & 65.2 & 67.2 & 67.5 & 66.3 \\
9 & 95.7 & 93.7 & 93.2 & 93.6 & 80.5 & 95.6 & 90.8 & 95.6 & 90.1 & 95.4 \\
10 & 85.7 & 83.1 & 82.9 & 81.7 & 68.9 & 85.0 & 81.9 & 85.2 & 81.0 & 85.1 \\
11 & 95.7 & 95.0 & 81.0 & 93.0 & 56.1 & 94.3 & 68.4 & 80.2 & 69.1 & 80.8 \\
12 & 85.4 & 84.1 & 71.8 & 82.0 & 53.8 & 80.5 & 62.1 & 72.3 & 64.4 & 72.1 \\
\hline
\end{tabular}

\subsection{Musk}

Musk is a well-known multi-instance data set [13]. Each data instance stands for a molecule, represented by a bag of all its possible conformations. A conformation is described by 166 numerical features. The molecules have to be classified as musk or non-musk. The data set consists of two parts. The first part contains 92 molecules, the second part 102. In each bag, there are between 2 and 40 conformations for the first part, and between 1 and 1044 for the second part.

Experiments were carried out using 10 -fold cross-validation. The simple methods have 5 hidden units and are trained for 500 iterations. For the cascadecorrelation networks, a pool of 20 neurons and 500 training iterations are used in every step. The data sets are a bit too small to use part of them as validation set. Therefore, the value of the correlation is used as stopping criterion. In the beginning, the correlation of a newly trained unit will be very high. This correlation decreases during the following training steps. Training will be stopped when the correlation falls below 0.75 .

The results for the musk data sets can be found in table 4. The best neural networks perform well compared with the other methods. They do not beat all of the multi-instance methods, but that is not unexpected given that these methods were specifically designed for multi-instance problems while these networks are more general. The cascade-correlation networks are all very small, in most cases with just one hidden unit. If one looks at the type of unit selected, then this is almost always a $\max$ or smx unit. This is the most logical choice in case of a multi-instance problem. The networks with this type of units also have the best performance for the simple networks. 
Table 4. Accuracies and 95\% confidence intervals for the musk data sets using 10 -fold crossvalidation. Results for other methods are obtained from [13].

\begin{tabular}{|l|c|c|}
\hline & musk 1 & musk 2 \\
\hline \hline iterated discrim APR & $92.4[87.0-97.8]$ & $89.2[83.2-95.2]$ \\
GFS elim-kde APR & $91.3[85.5-97.1]$ & $80.4[72.7-88.1]$ \\
GFS elim-count APR & $90.2[84.2-96.3]$ & $75.5[67.1-83.8]$ \\
GFS all-positive APR & $83.7[76.2-91.2]$ & $66.7[57.5-75.8]$ \\
all-positive APR & $80.4[72.3-88.5]$ & $72.6[63.9-81.2]$ \\
backpropagation & $75.0[66.2-83.8]$ & $67.7[58.6-76.7]$ \\
C4.5 (pruned) & $68.5[40.9-61.3]$ & $58.8[49.3-68.4]$ \\
\hline casc-corr & $85.3[83.1-87.5]$ & $75.5[72.6-78.4]$ \\
sym & $75.8[74.1-77.5]$ & $71.1[68.8-73.4]$ \\
lrc & $77.2[75.6-78.8]$ & $75.2[72.7-77.7]$ \\
frc & $74.9[73.4-76.4]$ & $73.3[70.8-75.8]$ \\
sum & $56.6[54.2-59.0]$ & $49.0[46.3-51.7]$ \\
hsum & $78.2[76.6-79.8]$ & $65.5[63.9-67.1]$ \\
max & $73.1[71.5-75.7]$ & $65.6[63.4-67.8]$ \\
hmax & $79.0[77.9-80.1]$ & $77.4[75.7-79.1]$ \\
smx & $72.2[71.0-73.4]$ & $62.8[60.8-64.8]$ \\
hsmx & $77.3[75.7-78.9]$ & $78.1[76.7-79.5]$ \\
\hline
\end{tabular}

\section{Conclusion}

In this paper, some subsymbolic approaches to learn aggregate function from bag data were discussed. These methods are based on neural networks. First, three simple methods were presented: adapted feedforward networks, recurrent networks and networks with special aggregation units integrated in the network. Another possibility is to combine these three approaches by using cascade-correlation, creating a method that automatically chooses the best of these options.

Results on artificial and multi-instance data sets were reported to assess the capacity of the different network structures. These results clearly show that it is possible to learn aggregate functions or at least approximate them with neural networks. They also indicate that the combined cascade-correlation approach is superior to any of the simple methods.

\section{References}

1. Blockeel, H., Bruynooghe, M.: Aggregation versus selection bias, and relational neural networks. In: Getoor, L., Jensen, D. (eds.) IJCAI 2003 Workshop on Learning Statistical Models from Relational Data, SRL 2003, Acapulco, Mexico (2003)

2. Krogel, M.A., Wrobel, S.: Transformation-based learning using multirelational aggregation. In: Rouveirol, C., Sebag, M. (eds.) ILP 2001. LNCS (LNAI), vol. 2157, pp. 142-155. Springer, Heidelberg (2001) 
3. Vens, C., Ramon, J., Blockeel, H.: Refining aggregate conditions in relational learning. In: Fürnkranz, J., Scheffer, T., Spiliopoulou, M. (eds.) PKDD 2006. LNCS (LNAI), vol. 4213, pp. 383-394. Springer, Heidelberg (2006)

4. Knobbe, A., Siebes, A., Marseille, B.: Involving aggregate functions in multirelational search. In: Principles of Data Mining and Knowledge Discovery, Proceedings of the 6th European Conference, August 2002, pp. 287-298. Springer, Heidelberg (2002)

5. Vens, C., Van Assche, A., Blockeel, H., Dzeroski, S.: First order random forests with complex aggregates. In: Camacho, R., King, R., Srinivasan, A. (eds.) ILP 2004. LNCS (LNAI), vol. 3194, pp. 323-340. Springer, Heidelberg (2004)

6. Van Assche, A., Vens, C., Blockeel, H., Dzeroski, S.: First order random forests: Learning relational classifiers with complex aggregates. Machine Learning 64(1-3), 149-182 (2006)

7. Uwents, W., Blockeel, H.: Classifying relational data with neural networks. In: Kramer, S., Pfahringer, B. (eds.) ILP 2005. LNCS (LNAI), vol. 3625, pp. 384-396. Springer, Heidelberg (2005)

8. Uwents, W., Blockeel, H.: Learning relational neural networks using a cascadecorrelation approach. In: Železný, F., Lavrač, N. (eds.) ILP 2008. LNCS (LNAI), vol. 5194, pp. 315-329. Springer, Heidelberg (2008)

9. Ramon, J., De Raedt, L.: Multi instance neural networks. In: Raedt, L.D., Kramer, S. (eds.) Proceedings of the ICML 2000 workshop on attribute-value and relational learning, pp. 53-60 (2000)

10. Fahlman, S.E., Lebiere, C.: The cascade-correlation learning architecture. In: Touretzky, D.S. (ed.) Advances in Neural Information Processing Systems, Denver, vol. 2, pp. 524-532. Morgan Kaufmann, San Mateo (1989)

11. Riedmiller, M., Braun, H.: A direct adaptive method for faster backpropagation learning: The RPROP algorithm. In: Proc. of the IEEE Intl. Conf. on Neural Networks, San Francisco, CA, pp. 586-591 (1993)

12. Michie, D., Muggleton, S., Page, D., Srinivasan, A.: To the international computing community: A new east-west challenge. Technical report, Oxford University Computing Laboratory, Oxford, UK (1994)

13. Dietterich, T.G., Lathrop, R.H., Lozano-Perez, T.: Solving the multiple instance problem with axis-parallel rectangles. Artificial Intelligence 89(1-2), 31-71 (1997) 